\title{
Advanced Glycosylation Products Quench Nitric Oxide and Mediate Defective Endothelium-dependent Vasodilatation in Experimental Diabetes
}

\author{
Richard Bucala, Kevin J. Tracey,* and Anthony Cerami \\ Laboratory of Medical Biochemistry, The Rockefeller University, New York 10021; and *Division of Neurosurgery, \\ Cornell University Medical College, New York 10021
}

\begin{abstract}
Nitric oxide (an endothelium-derived relaxing factor) induces smooth muscle relaxation and is an important mediator in the regulation of vascular tone. Advanced glycosylation end products, the glucose-derived moieties that form nonenzymatically and accumulate on long-lived tissue proteins, have been implicated in many of the complications of diabetes and normal aging. We demonstrate that advanced glycosylation products quench nitric oxide activity in vitro and in vivo. Acceleration of the advanced glycosylation process in vivo results in a time-dependent impairment in endothelium-dependent relaxation. Inhibition of advanced glycosylation with aminoguanidine prevents nitric oxide quenching, and ameliorates the vasodilatory impairment. These results implicate advanced glycosylation products as important modulators of nitric oxide activity and endothelium-dependent relaxation. (J. Clin. Invest. 1991. 87:432438.) Key words: endothelium-derived relaxing factor • glycation • aminoguanidine $\bullet$ hypertension
\end{abstract}

\section{Introduction}

An important mediator of smooth muscle responsiveness and resting vascular tone is endothelium-derived relaxing factor (1). Produced by endothelial cells in response to diverse humoral stimuli, this factor traverses the subendothelial space and activates smooth muscle cell guanylate cyclase. Intracellular cyclic guanosine monophosphate levels increase, leading to smooth muscle relaxation and vasodilatation. The active constituent of endothelium-derived relaxing factor recently has been identified to be the free radical species nitric oxide $(\mathrm{NO})^{1}$ $(2,3)$.

Interposed in the anatomic pathway of nitric oxide action is a layer of subendothelial collagen that separates the intimal endothelial cells from the smooth muscle cells of the media. Recent studies have demonstrated that vascular collagen accumulates in situ, through covalent, posttranslational modification, the products of advanced glycosylation (Fig. 1) $(4,5)$

Address reprint requests to Dr. Richard Bucala, Box 277, The Rockefeller University, 1230 York Avenue, New York, NY 10021.

Received for publication 18 June 1990 and in revised form $10 \mathrm{Au}$ gust 1990.

1. Abbreviations used in this paper: AFGP, 1-alkyl-2-formyl-3,4-diglycosylpyrrole; FFI-HA, 4-furanyl-2-furoyl-1 $\mathrm{H}$-imidazole-1-hexanoic acid; NO, nitric oxide.

J. Clin. Invest.

(c) The American Society for Clinical Investigation, Inc.

0021-9738/91/02/0432/07 \$2.00

Volume 87, February 1991, 432-438
These products represent the terminal adducts of the nonenzymatic glycation reaction between glucose and the amino groups of protein $(6,7)$. Because matrix collagen turns over very slowly, advanced glycosylation end products accumulate with age, and form at an accelerated rate when plasma glucose is elevated (as in diabetes mellitus) $(5,8,9)$.

Examination of the structures of model advanced glycosylation products (10-12) and of those compounds that have been isolated directly from tissue (13) led us to consider the possibility that these protein-bound moieties might react with and quench nitric oxide. Such an interaction would prevent endothelial cell-derived nitric oxide from activating smooth muscle relaxation. Interest in this possibility was motivated by observations that defective endothelium-dependent relaxation is a prominent feature in situations where high levels of advanced glycosylation end products occur, such as in diabetes (14-16) and in aging $(17,18)$.

In this study, we demonstrate that advanced glycosylation products inactivate nitric oxide via a rapid chemical reaction. The participation of advanced glycosylation products in nitric oxide quenching in vivo was examined in a rat model of experimentally induced diabetes. Chronic hyperglycemia accelerates the advanced glycosylation reaction and the deposition of advanced glycosylation end products $(5,8,9)$. Diabetic rats displayed a time-dependent impairment in vasodilatory responses to nitric oxide generating agents that was not reversed by insulin administration. The selective pharmacological inhibition of advanced glycosylation slowed the development of the vasodilatory impairment. These studies indicate that advanced glycosylation products are important modulators of endotheliumdependent relaxation, and that nitric oxide quenching is likely to play an important role in the evolution of vasodilatory defects in vivo.

\section{Methods}

Preparation of advanced glycosylation end products. Lysine-derived advanced glycosylation products were prepared by incubating $1 \mathrm{M}$ glucose-6-phosphate with $50 \mathrm{mM}$ L-lysine in $0.2 \mathrm{M}$ sodium phosphate buffer, $\mathrm{pH} \mathrm{7.4,} \mathrm{for} 10 \mathrm{~d}$. All incubations were performed sterilely, anaerobically, in the dark, and at $37^{\circ} \mathrm{C}$. Final products were observed to have an $A_{350}$ of $0.68 \mathrm{mM}^{-1}$ lysine equivalent. Albumin-derived advanced glycosylation products were prepared by incubating $50 \mathrm{mg} / \mathrm{ml}$ human serum albumin (type V; Sigma Chemical Co., St. Louis, MO) with 0.5 M glucose-6-phosphate or $1 \mathrm{M}$ glucose in $0.1 \mathrm{M}$ sodium phosphate buffer ( $\mathrm{pH} \mathrm{7.45)}$ for $8 \mathrm{wk}$ at $37^{\circ} \mathrm{C}$. Unreacted carbohydrate was removed before assay by extensive dialysis against PBS. Final derivitized products yielded an $\mathrm{A}_{350}$ of $45.9 \mathrm{mM}^{-1}$ albumin. Collagen type IV (human placental; Sigma Chemical Co.) was incubated with glucose as above except that the final protein concentration was $5 \mathrm{mg} / \mathrm{ml}$. For the time course studies (Fig. 6), test solutions were assayed without further dilution.

Albumin-derived advanced glycosylation products were reduced with sodium borohydride $\left(\mathrm{NaBH}_{4}\right)$ by adding a 200 -fold molar excess 


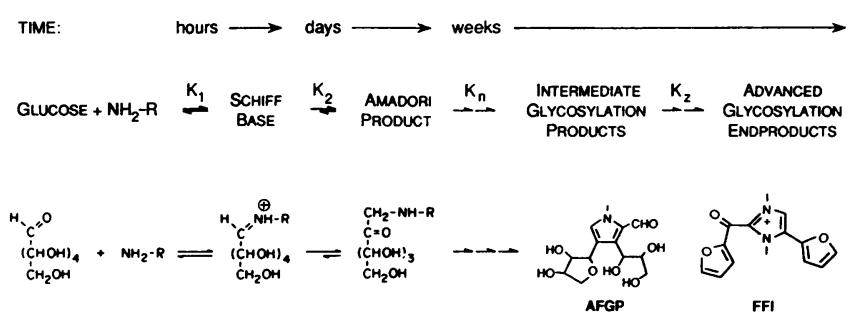

Figure 1. Schematic conception of the time course of the formation of early, intermediate, and advanced glycosylation products. $\mathrm{NH}_{2}-\mathrm{R}$ denotes either the $\alpha$ - or $\epsilon$-amino groups of protein (R). The structures of glucose-derived products discussed in this study are shown. $A F G P$ and $F F I$ are model compounds representative of intermediate and advanced glycosylation products, respectively.

of borohydride to albumin (19). The mixture was incubated at room temperature for $10 \mathrm{~min}$, on ice for $50 \mathrm{~min}$, and then dialyzed extensively. Aminoguanidine hydrochloride (Aldrich Chemical Co., Milwaukee, WI) was added at the initiation of the advanced glycosylation reactions. 1-Deoxy-1-morpholino-D-fructose was obtained from Sigma and 1-deoxy-1-propylamino-D-fructose was prepared from $\alpha$-D-glucose and $n$-propylamine following the method of Michel and Hagemann (20). The model advanced glycosylation product 4-furanyl-2-furoyl$1 \mathrm{H}$-imidazole-1-hexanoic acid (FFI-HA) was synthesized from an aqueous mixture of furylglyoxal and 6-aminohexanoic acid and purified by medium pressure chromatography on silica gel as described previously (21). Glucose-derived 1-alkyl-2-formyl-3,4-diglycosylpyrrole (AFGP) was prepared by incubating glucose with 6-aminohexanoic acid and sodium sulfite for $26 \mathrm{~d}$ at $37^{\circ} \mathrm{C}$ followed by chromatography on Dowex AG $1 \times 4$ anion exchange resin and FPLC (11). Structures and purity of synthesized compounds were confirmed by proton nuclear magnetic resonance spectroscopy $(10,11)$.

Nitric oxide assay. Nitric oxide activity was measured chemically following the modifications (2) of a procedure described by Bell et al. (22). This method relies on the diazotization of sulfanilic acid by nitric oxide at acid $\mathrm{pH}$ and subsequent coupling to $N$-(1-naphthyl)-ethylenediamine. Colored product at an OD of $540 \mathrm{~nm}$ forms quantitatively in the presence of nitric oxide or compounds that spontaneously release nitric oxide. For these studies, a saturated solution (1-2 mM) of authentic nitric oxide solution was prepared by bubbling nitric oxide gas (Matheson Gas Products, Inc., Bridgeport, NJ) for 2 min into ice-cold, deoxygenated $\mathrm{H}_{2} \mathrm{O}$ under anaerobic conditions in sealed, gas-tight vials (2). Although the diazotization reaction is specific for nitric oxide (NO), nitrite $\left(\mathrm{NO}_{2}^{-}\right)$, an oxidation product of nitric oxide is detected at a many-fold lower sensitivity $(2,22)$. This was confirmed in our study by observing a marked diminution in absorbance after aeration of the nitric oxide standard solution. Nitric oxide quenching was determined by incubating anaerobically an aliquot $(50 \mu \mathrm{l})$ of nitric oxide solution (yielding a final concentration of $5 \mu \mathrm{M}$ ) with the test solution $(0.2 \mathrm{ml}$ ) for $5 \mathrm{~min}$. At the end of this time, $0.1 \mathrm{ml}$ of a solution of $3.75 \mathrm{M} \mathrm{HCl}$ and $18.75 \mathrm{M}$ sulfanilic acid was added, followed by the addition of 0.05 $\mathrm{ml}$ of $12.5 \mathrm{M}$ ethylenediamine. Absorbance values were recorded after an additional $10 \mathrm{~min}$ of incubation at room temperature. The addition of glycosylation products to the test assay in any other sequence did not affect the measurement of nitric oxide, indicating that the quenching effect of these compounds was due to reaction with nitric oxide, and not the result of interference in the assay for nitric oxide. Quenching values were calculated after subtraction of reagent blanks (without nitric oxide addition) and represent duplicate measurements of at least two experiments.

Animal studies. Male Lewis rats (Charles River Breeding Laboratories, Inc., Wilmington, MA) aged $10 \mathrm{wk}$ were used in these studies which were conducted in accordance with The Rockefeller University Laboratory Animal Research Center guidelines. After a 1-wk adaptation period, diabetes was induced in rats by rapid intravenous injection of streptozotocin $(65 \mathrm{mg} / \mathrm{kg}$ ) (Sigma Chemical Co.) and confirmed by measurement of serum glucose at $2 \mathrm{wk}(480 \pm 42 \mathrm{mg} \%)$ and at $4 \mathrm{wk}$. Urinary ketones were measured with Ketostix (Miles Ames Div., Miles Laboratories, Inc., Elkhart, IN).

For blood pressure studies, rats were anesthetized with $50 \mathrm{mg} / \mathrm{kg}$ i.p. Nembutal and the common carotid artery surgically exposed and cannulated with polyethylene tubing (PE 50). Blood pressure, heart rate, and respiratory rate were monitored continuously with a pressure transducer and a model 7702B recorder (Hewlett-Packard Co., Palo Alto, CA). Dose-response curves for acetylcholine and nitroglycerin were generated by injecting drugs through a three-way stopcock directly into the ascending aorta. Drugs were diluted in sterile $\mathrm{H}_{2} \mathrm{O}$ to produce a constant injectable volume of $1 \mathrm{ml} \mathrm{kg}^{-1}$ animal weight. The hypotensive response was measured at $1 \mathrm{~min}$ postinjection and the percent change in mean arterial pressure calculated. Increasing doses of drugs were then injected in each animal after at least a $4 t_{1 / 2}$ washout from the prior injection. Baseline blood pressure and heart rate were not observed to vary significantly among the different experimental groups. Duplicate measurements for each drug dose were obtained for each experimental animal. Animals were not reused poststudy. In addition, one group of diabetic animals $(n=4$, duration of diabetes $2 \mathrm{mo}$ ) was administered subcutaneous insulin for $96 \mathrm{~h}$ ( $2 \mathrm{U} \mathrm{NPH}$ insulin/100 $\mathrm{mg}$ body weight bidaily).

The aminoguanidine-treated, diabetic group was administered 250 $\mathrm{mg} / \mathrm{kg}$ aminoguanidine hydrochloride (dissolved in sterile $\mathrm{H}_{2} \mathrm{O}$ ) by single daily oral dose, beginning $7 \mathrm{~d}$ before the induction of diabetes. Both treated and untreated diabetic groups manifested similar levels of hyperglycemia (diabetic group: $480 \pm 42 \mathrm{mg} \%$, aminoguanidine treated group: $468 \pm 43 \mathrm{mg} \%$ ). In separate experiments, control (nondiabetic) rats $(n=4)$ also were administered aminoguanidine and studied for blood pressure responses.

\section{Results}

Advanced glycosylation products inactivate nitric oxide in vitro. We initially observed the quenching of nitric oxide by advanced glycosylation products in the following experimental system. Model advanced glycosylation products were prepared by incubating lysine with glucose-6-phosphate for $10 \mathrm{~d}$ at $37^{\circ} \mathrm{C}$. Glucose-6-phosphate was chosen as a model sugar because its rate of anomerization favors the open-chain form. This accelerates the initial condensation reaction between the sugar and primary amino groups (23). High concentrations of sugar also accelerate advanced glycosylation. These incubation conditions yield fluorescent, glucose-derived lysine adducts that possess the physicochemical and spectral properties of advanced glycosylation products that are bound to protein in vivo (24). As shown in Fig. 2 (top) the addition of increasing amounts of lysine-derived advanced glycosylation products to nitric oxide results in a first-order inactivation curve for nitric oxide. A control experiment in which lysine and glucose-6-phosphate were allowed to incubate alone, followed by mixing and incubation with nitric oxide, did not yield products with quenching activity. Time course studies showed that the quenching reaction occurred rapidly ( $<5 \mathrm{~s}$ to completion, data not shown).

Advanced glycosylation products that are bound to protein also quench nitric oxide. Fig. 1 (bottom) shows the nitric oxidequenching effect of human serum albumin that had been incubated with glucose-6-phosphate and then dialyzed to remove unbound products. Similar first-order quenching kinetics were observed. Unmodified albumin (incubated under the same conditions, but without sugar) showed no measurable quenching activity. 

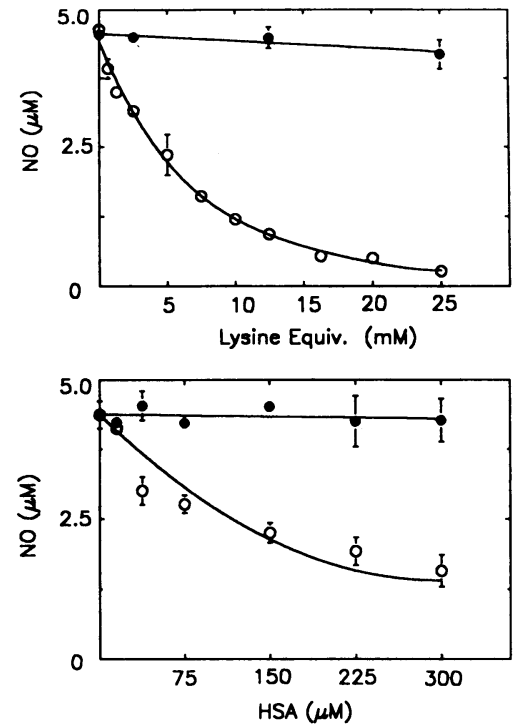

Figure 2. Quenching of nitric oxide by advanced glycosylation products in vitro. (Top) Addition of increasing amounts of lysine-derived advanced glycosylation products to NO (o). Addition of equivalent amounts of lysine and glucose-6-phosphate that were incubated under identical conditions but separated until the quenching assay (๑). (Bottom) Addition of increasing amounts of human serum albumin modified by advanced glycosylation (o), and albumin incubated

under the same conditions but without sugar $(\bullet)$. Values express the mean \pm SEM of duplicate measurements obtained in two independent experiments. Points without error bars represent a SEM $<5 \%$.

Advanced glycosylation products prepared by the incubation of sugars with either lysine or albumin consist of a mixture of products that represent many intermediates in the advanced glycosylation pathway $(7,25)$. To acquire some information about the structural requirements of quenching activity, lysine and albumin-derived advanced glycosylation products were subjected to chemical modification and reassayed for quenching activity (Fig. 3). Reduction with sodium borohydride did not affect nitric oxide quenching, suggesting that the quenching species was not an early glycosyl adduct, such as a Schiff base, or the Amadori product $(7,25)$. It is significant that the addition of aminoguanidine ( $400 \mathrm{mM})$ to the albumin/glucose-6-phosphate incubation inhibited the formation of the quenching species. Aminoguanidine is a hydrazine-like compound that inhibits the progression of advanced glycosylation both in vitro and in vivo $(26,27)$.

Two model advanced glycosylation end products were synthesized de novo and tested for their ability to quench nitric

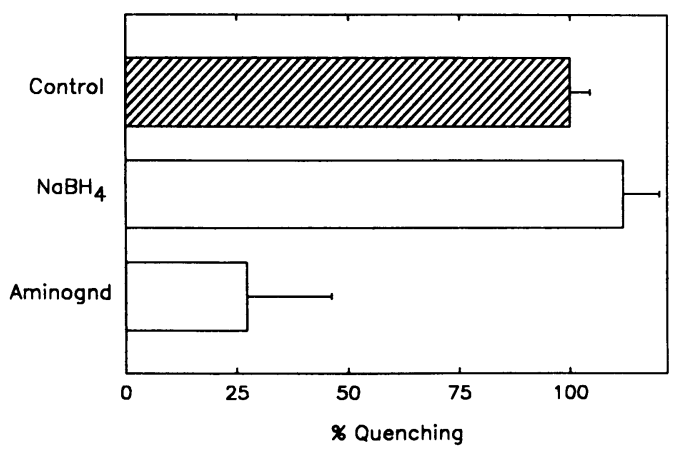

Figure 3. Effect of chemical modification on quenching activity. The percent decrease in NO quenching was compared with a control amount of advanced glycosylation products that was added to quench nitric oxide by $50 \%$ ( $40 \mathrm{nmol}$ of albumin-derived products). Aminognd: aminoguanidine. Error bars represent the SEM of duplicate measurements in two separate experiments.

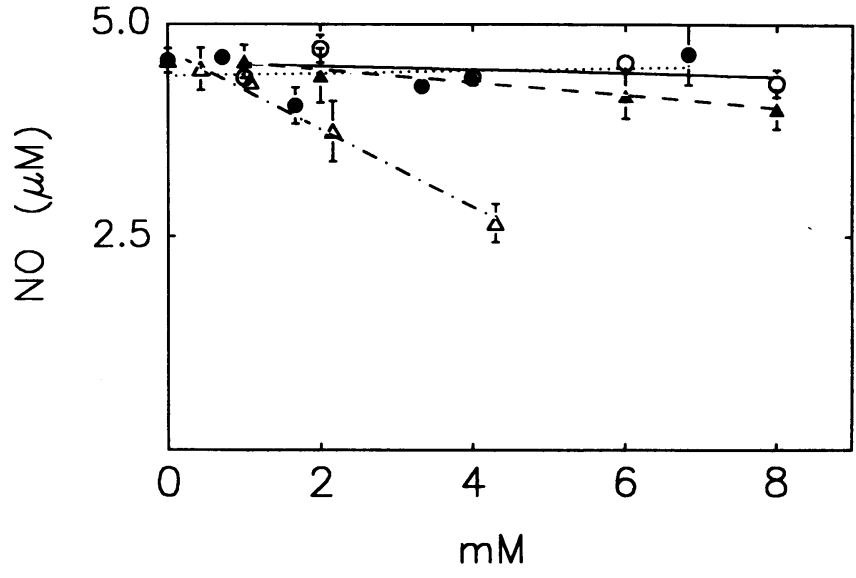

Figure 4. The effect of increasing amounts of representative glycosylation products on nitric oxide quenching.

Deoxymorpholinofructose (A), deoxypropylaminofructose (o), FFIHA (๑), and AFGP $(\Delta)$. Error bars represent the SEM of duplicate measurements in two independent experiments.

oxide (Fig. 4). The compound AFGP (11) displayed quenching activity. The compound FFI-HA $(10,21)$ did not quench nitric oxide within the concentration range tested. AFGP is believed to represent an intermediate in the advanced glycosylation pathway because it was isolated under conditions in which the terminal steps of advanced glycosylation were inhibited (11). Fig. 4 also shows the lack of quenching activity of two Amadori products, deoxymorpholinofructose and deoxypropylaminofructose.

The effect of aminoguanidine on the formation of the nitric oxide-quenching species was studied further in a time course of both the advanced glycosylation reaction and nitric oxide quenching. Fig. 5 shows that the time-dependent formation of advanced glycosylation products (measured by increasing opti-

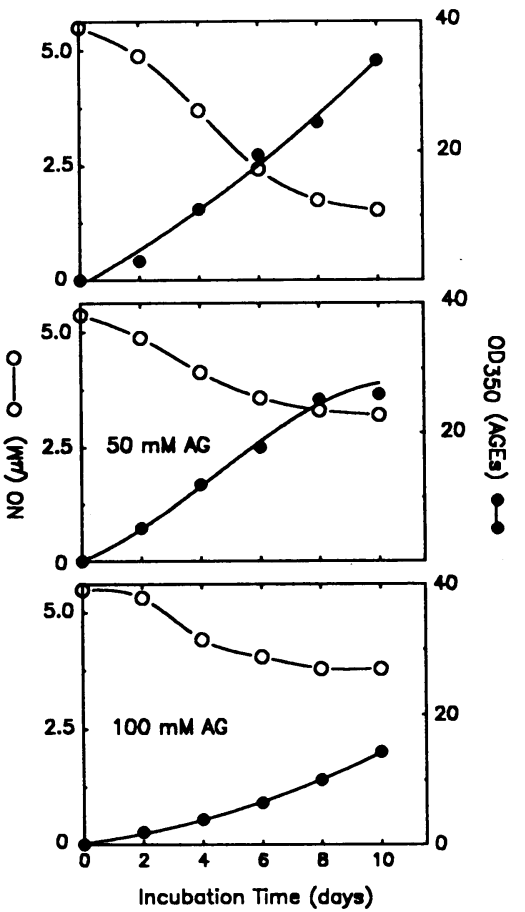

Figure 5. Time course for the formation of nitric oxide-quenching species and effect of aminoguanidine. Formation of lysinederived advanced glycosylation products (๑) and nitric oxide quenching activity (o) Incubations contained no aminoguanidine (top), $50 \mathrm{mM}$ aminoguanidine (middle), and $100 \mathrm{mM}$ aminoguanidine (bottom). Advanced glycosylation was measured by increasing absorbance at $\mathrm{OD}_{350}$. Points represent the mean of duplicate determinations. Data shown are representative of two experiments. 
cal absorbance at $350 \mathrm{~nm}$ ) led to the time-dependent production of a quenching product. It is interesting that the kinetics of quenching product formation did not precisely parallel the formation of the characteristic absorbance changes of advanced glycosylation. Quenching activity formed rapidly during days 2-6, whereas advanced glycosylation was linear during this period. It appears that the quenching product forms after the Amadori product, but still relatively early in the advanced glycosylation pathway. Although quenching activity may result from the interaction of nitric oxide with AFGP or a related product, a radical-mediated inactivation reaction may also occur. Electron spin resonance studies have shown that free radical species form during the early phase of advanced glycosylation (28). Fig. 5 also demonstrates that the inhibitory effect of aminoguanidine is concentration dependent, i.e., addition of increasing amounts of aminoguanidine inhibits both advanced glycosylation and quenching product formation to an increasing degree.

The time-dependent modification of human serum albumin and collagen by glucose also led to the time-dependent formation of a nitric oxide-quenching species (Fig. 6). Aminoguanidine inhibited both advanced glycosylation and nitric oxide quenching. The increased rate of advanced glycosylation

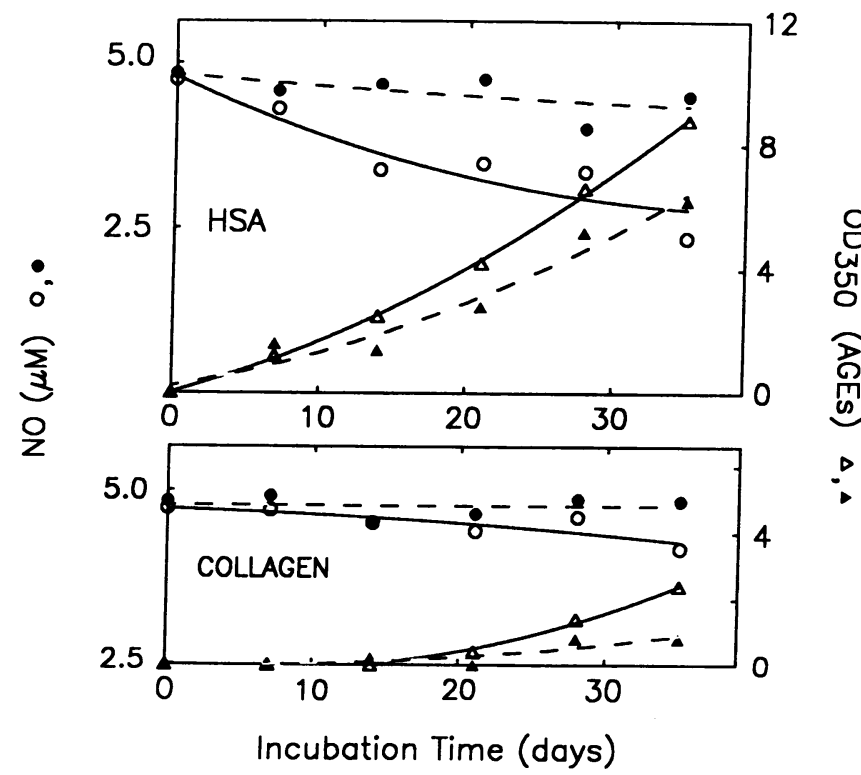

Figure 6. Correlation between the formation of protein-bound advanced glycosylation products and nitric oxide quenching, and inhibition of both processes with aminoguanidine. Human serum albumin $(50 \mathrm{mg} / \mathrm{ml})$ or collagen type IV $(5 \mathrm{mg} / \mathrm{ml})$ was incubated with $1 \mathrm{M}$ glucose in $0.1 \mathrm{M}$ sodium phosphate buffer $\left(\mathrm{pH} \mathrm{7.45)}\right.$ at $37^{\circ}$ and then dialyzed to remove unbound products. Nitric oxide quenching activity was assayed without further dilution. (Top) Formation of glucose-derived advanced glycosylation products on human serum albumin $(H S A)(\Delta)$, and inhibition with aminoguanidine $(200 \mathrm{mM})(\triangle)$. Formation of albumin-bound nitric oxide quenching moieties (o), and inhibition with aminoguanidine $(\bullet)$. (Bottom) Formation of glucose-derived advanced glycosylation products on collagen $(\Delta)$, and inhibition with aminoguanidine $(200$ $\mathrm{mM})(\triangle)$. Formation of collagen-bound nitric oxide quenching moieties (o), and inhibition with aminoguanidine (๑). Each point represents the mean of duplicate measurements and data are representative of two independent experiments. and quenching product formation with albumin versus collagen reflects in part the decreased amount of collagen present in the incubation (performed with soluble collagen type IV, at a solubility limit in neutral buffer of $5 \mathrm{mg} / \mathrm{ml}$ ).

Advanced glycosylation products inhibit nitric oxide-mediated vasodilatation. We reasoned that the advanced glycosylation products that form in vivo might alter the vascular response to pharmacological agents that act via a nitric oxide intermediate. To examine this possibility, we studied the blood pressure responses of experimental rats to the vasodilating agents acetylcholine and nitroglycerin. Acetylcholine is an endothelium-dependent agent that stimulates the endothelial cell to produce nitric oxide via a receptor-mediated mechanism (29), whereas nitroglycerin is an endothelium-independent agent that generates nitric oxide directly and nonenzymatically (30). Since long-term hyperglycemia accelerates protein glycation and the subsequent accumulation of advanced glycosylation products on matrix and vascular wall collagen $(5,8,9)$, one group of animals was subjected to chronic hyperglycemia by inducing the diabetic state with streptozotocin. At various time intervals after the induction of diabetes, intraarterial cannulas were inserted and the vasodilatory response to increasing doses of acetylcholine and nitroglycerin measured. The hyperglycemic animals were found to have an impaired endothelium-dependent (acetylcholine) relaxation response that increased with the duration of hyperglycemia (Fig. 7, top). Peak impairment occurred at $2 \mathrm{mo}$, and equaled the degree of impairment that was observed in animals made diabetic $1 \mathrm{yr}$ previously. A similar time-dependent impairment in vasodilatation was observed with the endothelium-independent agent nitroglycerin (Fig. 7, bottom). The observation that impaired vasodilatation occurred in response to both endothelial cellderived nitric oxide and to chemically generated nitric oxide suggested that the vasodilatory defect in these animals must be a postendothelial cell phenomenon.

To assess the contribution of hyperglycemic physiology to vascular dilatation, one group of animals ( $n=4$, duration of diabetes $2 \mathrm{mo}$ ) was administered subcutaneous insulin for $4 \mathrm{~d}$ and then studied for impaired vasodilatation. As expected, within $72 \mathrm{~h}$ these animals were found to have blood glucose in the $120-180 \mathrm{mg} \%$ range and to have had a reversal in ketogenesis (assessed by the absence of urinary ketones). Dose-response curves for acetylcholine and nitroglycerin demonstrated only a minor improvement in the vasodilatory response; with the dose-response curve for the insulin treated group falling between the 2 mo and the 1-mo diabetes duration group (data not shown).

Taken together, these observations suggest that the impaired vasodilatatory response in experimental diabetes is the result of a long-term, postendothelial cell effect. This is consistent with the excessive accumulation of advanced glycosylation products within the subendothelial collagen. To address directly the role of advanced glycosylation in nitric oxide quenching, one group of hyperglycemic rats was administered the advanced glycosylation inhibitor, aminoguanidine. Previous studies in experimentally induced diabetes have demonstrated that aminoguanidine significantly prevents the accumulation of advanced glycosylation end products both in vascular wall collagen and in long-lived basement membrane proteins $(26,27)$. A control study of vasodilatation in normal rats $(n=4)$ revealed that aminoguanidine administration does not affect baseline vascular responses when these animals were 

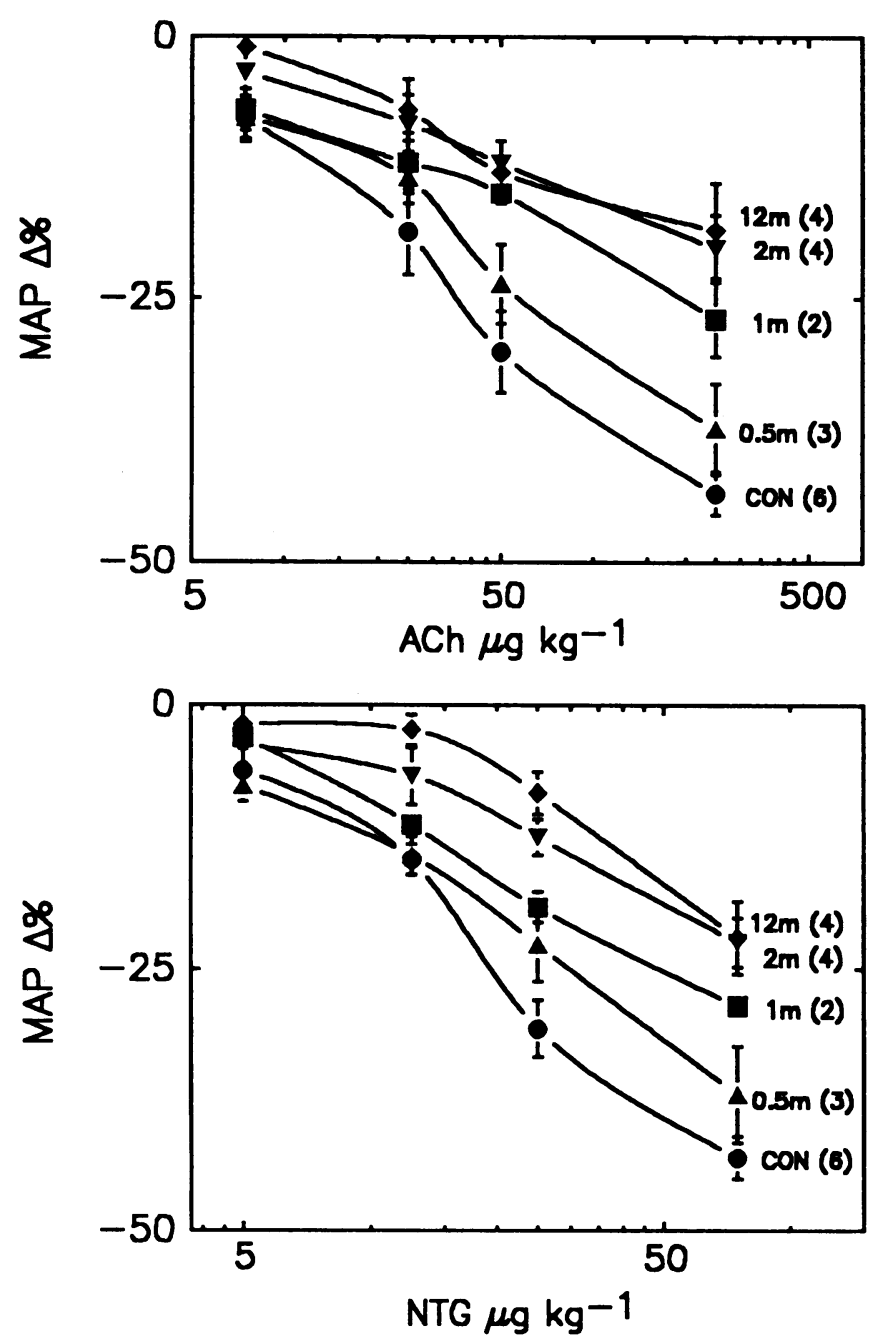

Figure 7. Time-dependent progression of vasodilatory impairment in rats with experimentally induced diabetes. (Top) Vasodilatory response to increasing doses of acetylcholine. (Bottom) Vasodilatory response to increasing doses of nitroglycerin. MAP: mean arterial pressure; $C O N$ : control; $m$ : duration of diabetes in months; numbers in parentheses, number of animals studied.

compared with an age-matched, untreated group (data not shown). Fig. 8 illustrates that when administered to diabetic rats, aminoguanidine had a significant sparing effect in the progression of the vasodilatory impairment. This effect was apparent at $1 \mathrm{mo}$ and became more pronounced after 2 mo of diabetes. The observation that the protective effect of aminoguanidine occurred in the case of both endothelium-dependent (acetylcholine) and endothelium-independent (nitroglycerin) agents is consistent with nitric oxide quenching at the subendothelial level. In an additional study, rats were administered aminoguanidine after diabetes had been established for 1 mo (when defective vasodilatation appears [Fig. 7]). In this case, after 2 mo of treatment with aminoguanidine, the vasodilatory responses of these animals $(n=4)$ did not differ significantly from the untreated diabetic group. This result further suggests that the action of aminoguanidine is to prevent the formation of glycosylation products that can quench nitric oxide. Once quenching intermediates form, i.e., after 1 mo of experimentally induced diabetes, aminoguanidine is ineffective in ameliorating the vasodilatory defect that occurs as a result of chronic hyperglycemia.

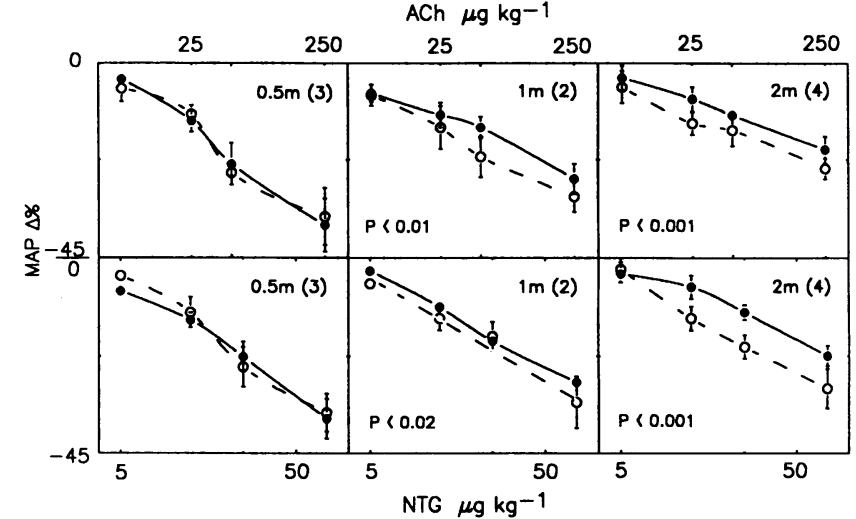

Figure 8. Time-dependent progression of vasodilatory impairment and effect of the inhibition of advanced glycosylation. Vasodilatory response to acetylcholine (top row) and nitroglycerin (bottom row) at $0.5 \mathrm{mo}(0.5 \mathrm{~m}$, left two panels $), 1 \mathrm{mo}(1 \mathrm{~m}$, middle two panels $)$, and 2 mo ( $2 \mathrm{~m}$, right two panels). (๑): diabetic animals; (o): diabetic animals treated with aminoguanidine. MAP, mean arterial pressure; numbers in parentheses, number of experimental animals studied for each drug. Statistical significance was calculated from the Student's $t$ test (two-tailed). The $P$ value within each panel compares the aminoguanidine-treated group vs. the untreated group for each drug at $1 \mathrm{mo}$ and at $2 \mathrm{mo}$

\section{Discussion}

The identification of nitric oxide as a mediator of endotheliumdependent relaxation has opened new avenues of investigation into both normal and abnormal vascular physiology (29). Defective endothelium-dependent relaxation characterizes many pathophysiological states, including diabetes (14-16), aging $(17,18)$, atherosclerosis $(31,32)$, and some forms of hypertension $(33,34)$. This study demonstrates that nitric oxide activity is modulated in part by acquired protein modifications that occur within the vascular wall. Advanced glycosylation is a posttranslational modification process that results in the formation of glucose-derived heterocycles with diverse chemical structures $(6,7,25)$. This process occurs ubiquitously on extracellular matrix proteins and advanced glycosylation products accumulate progressively with age. This study demonstrates that relatively early in the advanced glycosylation pathway, reactive intermediate(s) form that can react with and quench nitric oxide. Quenching occurs rapidly $(<5 \mathrm{~s})$ and appears to result from a direct reaction between the nitric oxide radical and the advanced glycosylation product(s). Time course and borohydride reduction studies indicate that the quenching product occurs after Amadori product formation. In vitro, formation of the quenching product plateaus while the characteristic absorbance and fluorescence changes of advanced glycosylation continue at a rapid rate. Presumably, as reactive, oxidizing moieties form within advanced glycosylation products, they become available for reaction with nitric oxide. It is significant that the time course of quenching product formation is similar to that which has been observed for the formation of free radicals during the advanced glycosylation reaction (28). Thus, a direct free radical reaction may also contribute to nitric oxide inactivation. Of two synthetic advanced glycosylation products that were tested for quenching activity, the compound AFGP, which represents an advanced glycosylation intermediate, was found to quench nitric oxide in vitro. Whether 
in vivo quenching activity is due to AFGP alone, or to other advanced glycosylation intermediates, must await further investigation.

Experimental rats with chronic hyperglycemia and accelerated advanced glycosylation end product deposition demonstrate a slow, time-dependent decrease in nitric oxide responsiveness. This vasodilatory impairment was not corrected by insulin administration and normalization of metabolic parameters such as blood glucose and ketones. The observation that defective vasodilatation occurred in response to both endothelium-dependent and endothelium-independent agents is consistent with nitric oxide inactivation by advanced glycosylation products within the subendothelial space. The role of advanced glycosylation in nitric oxide inactivation in vivo was confirmed by administering the advanced glycosylation inhibitor aminoguanidine to diabetic animals. In these animals a significant amelioration in the vasodilatory impairment was observed.

The impairment in the vasodilatory response was found to plateau at $2 \mathrm{mo}$, and equaled the magnitude of the defect present after $1 \mathrm{yr}$ of diabetes (Fig. 7). The time course of vasodilatory impairment is consistent with the formation of post-Amadori glycosylation products that form over a period of several weeks in experimental diabetes (26). Structural studies of collagen modification have demonstrated that in both normal and diabetic individuals, advanced glycosylation end products continue to accumulate over many years $(5,8,9)$. Thus the "plateauing" of vasodilatory impairment may be due to additional factors that limit the progression of the vasodilatory defect. Other hemodynamic controls, such as prostaglandin (35), catecholamine (36), or electrolyte (37) mediated pathways may supervene and prevent further impairment in vasodilatation. An alternative explanation for the plateauing effect would be that formation of the nitric oxide quenching moiety becomes saturated after 2 mo of hyperglycemia. This is supported by the in vitro quenching data (Fig. 5) that indicate that the formation of the quenching intermediate plateaus while advanced glycosylation continues at a rapid rate. In vivo this situation would be analogous to the accumulation of the fructosyl-lysine Amadori product on long-lived structural proteins. Tissue measurements, in agreement with calculated equilibrium constraints, have demonstrated that Amadori product formation always plateaus at a level that is 3-3.5-fold higher than normal, irrespective of the absolute duration of hyperglycemia $(38,39)$.

Defective endothelium-dependent relaxation is a feature of both experimental and human diabetes (14-16). The results of recent animal $(40,41)$ and human studies $(42)$ indicate that nitric oxide is produced tonically by endothelial cells, implicating it as an important mediator of normal resting vascular tone. The time-dependent inactivation of nitric oxide by advanced glycosylation end products may represent an important mechanism in the evolution of diabetes and age-related hypertension. The existence of animal models for accelerated advanced glycosylation, as well as specific pharmacological inhibitors of both advanced glycosylation and nitric oxide production, will facilitate the future study of this process.

Recent studies have expanded the role of nitric oxide from endothelium-dependent relaxation to that of an intercellular messenger between neurons (43), hepatic cells (44), and within the kidney mesangium (45). Advanced glycosylation products form ubiquitously on extracellular matrix proteins and have been implicated in many of the physicochemical abnormalities that characterize diabetic and aged proteins $(9,46)$, including increases in cross-linking and decreases in chemical and proteolytic digestibility. This study indicates that advanced glycosylation products also play a role in the modulation of cellular communication, suggesting that nitric oxide quenching may contribute to the hyperglycemic and age-associated changes that occur on the multicellular and organismal level.

\section{Acknowledgments}

We are grateful to Drs. P. Ulrich, N. Murgolo, and C. Chen for assistance with the Amadori product and AFGP and FFI structural studies. We thank Ms. L. Zeltman and the Laboratory Animal Research Center at The Rockefeller University for assistance with the animal experiments. The technical assistance of Ms. L. Chiu is also gratefully acknowledged.

R. Bucala is a Brookdale National Fellow. This work was supported by a grant from the American Diabetes Association.

\section{References}

1. Furchgott, R. F. 1984. The role of endothelium in the responses of vascular smooth muscle to drugs. Annu. Rev. Pharmacol. Toxicol. 24:175-197.

2. Ignarro, L. J., G. M. Buga, K. S. Wood, R. E. Byrns, and G. Chaudhuri. 1987. Endothelium-derived relaxing factor produced and released from artery and vein is nitric oxide. Proc. Natl. Acad. Sci. USA. 84:9265-9269.

3. Palmer, R. M. J., A. G. Ferrige, and S. Moncada. 1987. Nitric oxide release accounts for the biological activity of endothelium-derived relaxing factor. $\mathrm{Na}$ ture (Lond.). 327:524-526.

4. Tanzer, M. L., R. Fairweather, and P. M. Gallop. 1972. Collagen crosslinks: isolation of $\mathrm{N}-\mathrm{\epsilon}$-hexosyl hydroxylysine from borohydride-reducible calf-skin insoluble collagen. Arch. Biochem. Biophys. 151:137-141.

5. Monnier, V. M., R. R. Kohn, and A. Cerami. 1984. Accelerated age-related browning of human collagen in diabetes mellitus. Proc. Natl. Acad. Sci. USA. 81:583-587.

6. Brownlee, M., H. Vlassara, and A. Cerami. 1984. Nonenzymatic glycosylation and the pathogenesis of diabetic complications. Ann. Intern. Med. 101:527537.

7. Njoroge, F. G., and V. M. Monnier. 1989. The chemistry of the Maillard reaction under physiological conditions: a review. Prog. Clin. Biol. Res. 304:85107.

8. Schnider, S. L., and R. R. Kohn. 1980. Glucosylation of human collagen in aging and diabetes mellitus. J. Clin. Invest. 66:1179-1181.

9. Monnier, V. M., V. Vishwanath, K. E. Frank, C. A. Elmets, P. Dauchot, and R. R. Kohn. 1986. Relation between complications of Type I diabetes mellitus and collagen-linked fluorescence. N. Engl. J. Med. 314:403-408.

10. Pongor, S., P. C. Ulrich, F. A. Bencsath, and A. Cerami. 1984. Aging of proteins: isolation and identification of a fluorescent chromophore from the reaction of polypeptides with glucose. Proc. Natl. Acad. Sci. USA. 81:2684-2688.

11. Farmar, J., P. Ulrich, and A. Cerami. 1988. Novel pyrroles from sulfite-inhibited Maillard reactions: insight into the mechanism of inhibition. J. Org. Chem. 53:2346-2349.

12. Njoroge, F. G., L. M. Sayre, and V. M. Monnier. 1987. Detection of D-glucose derived pyrrole compounds during Maillard reaction under physiological conditions. Carbohydr. Res. 167:211-220.

13. Sell, D. R., and V. M. Monnier. 1989. Structure elucidation of a senescence cross-link from human extracellular matrix. J. Biol. Chem. 264:2159721602 .

14. Oyama, Y., H. Kawasaki, Y. Hattori, and M. Kanno. 1986. Attenuation of endothelium-dependent relaxation in aorta from diabetic rats. Eur. J. Pharma col. 131:75-78.

15. Meraji, S., L. Jayakody, M. P. J. Senaratne, A. B. R. Thomson, and T. Kappagoda. 1987. Endothelium-dependent relaxation in aorta of BB rat. Diabetes. 36:978-981.

16. de Tejada, I. S., I. Goldstein, K. Azadzoi, R. J. Krane, and R. J. R. Cohen. 1989. Impaired neurogenic and endothelium-mediated relaxation of penile smooth muscle in diabetic men with impotence. N. Engl. J. Med. 320:10251030.

17. Brink, C., P. G. Duncan, and J. S. Douglas. 1984. Decreased vascular sensitivity to histamine during aging. Agents Actions. 14:8-10.

18. Gascho, J. A., C. Fanelli, and R. Zelis. 1989. Aging reduces venous distensibility and the venodilatory response to nitroglycerin in normal subjects. $\mathrm{Am}$. J. Cardiol. 63:1267-1270.

19. Fluckiger, R., and P. M. Gallop. 1984. Measurement of nonenzymatic protein glycosylation. Methods Enzymol. 106:77-87. 
20. Michel, F., and G. Hagemann. 1959. Darstellung apliphatischer AmadoriProdukte. Chem. Ber. 92:2836-2840.

21. Chang, J. C. F., P. Ulrich, R. Bucala, and A. Cerami. 1985. Detection of an advanced glycosylation product bound to protein in situ. J. Biol. Chem. 260:7070-7074.

22. Bell, F. K., J. J. O'Neill, and R. M. Burgison. 1963. Determination of the oil/water distribution coefficients of glyceryl trinitrate and two similar nitrate esters. J. Pharm. Sci. 52:637-639.

23. Schwimmer, S., and H. S. Olcott. 1953. Reaction between glycine and hexose phosphates. J. Am. Chem. Soc. 75:4855.

24. Monnier, V. M., and A. Cerami. 1981. Nonenzymatic browning in vivo: possible process for aging of long-lived proteins. Science (Wash. DC). 211:491493.

25. Ledl, F., J. Beck, M. Sengl, H. Osiander, S. Estendorfer, T. Severin, and B. Huber. 1989. Chemical pathways of the Maillard reaction. Prog. Clin. Biol. Res. 304:23-42.

26. Brownlee, M., H. Vlassara, T. Kooney, P. Ulrich, and A. Cerami. 1986. Aminoguanidine prevents diabetes-induced arterial wall protein cross-linking. Science (Wash. DC). 232:1629-1632.

27. Nicholls, K., and T. E. Mandel. 1989. Advanced glycosylation endproducts in experimental murine diabetic nephropathy: effect of islet isografting and aminoguanidine. Lab. Invest. 60:486-491.

28. Namiki, M., and T. Hayashi. 1981. Formation of novel free radical products in an early stage of Maillard reaction. Prog. Food \& Nutr. Sci. 5:81-91.

29. Ignarro, L. J. 1990. Biosynthesis and metabolism of endothelium-derived nitric oxide. Annu. Rev. Pharmacol. Toxicol. 30:535-560.

30. Feelisch, M., and E. A. Noack. 1987. Correlation between nitric oxide formation during degradation of organic nitrates and activation of guanylate cyclase. Eur. J. Pharmacol. 139:19-30.

31. Bossaller, C., G. B. Habib, H. Yamamoto, C. Williams, S. Wells, and P. D. Henry. 1987. Impaired muscarinic endothelium-dependent relaxation and cyclic guanosine 5 '-monophosphate formation in atherosclerotic human coronary artery and rabbit aorta. J. Clin. Invest. 79:170-174.

32. Ludmer, P. L., A. Selwyn, T. L. Shook, R. R. Wayne, G. H. Mudge, R. W Alexander, and P. Ganz. 1986. Paradoxical vasoconstriction produced by acetylcholine in atherosclerotic coronary arteries. N. Engl. J. Med. 315:1046-1051.

33. Lockette, W., Y. Otsuka, and O. Carretero. 1986. The loss of endothelium-dependent vascular relaxation in hypertension. Hypertension (Dallas). 8:II61-II-66.
34. Panza, J. A., A. A. Quyyumi, J. E. Brush, and S. E. Epstein. 1990. Abnormal endothelium-dependent vascular relaxation in patients with essential hypertension. N. Engl. J. Med. 323:22-27.

35. Tesfamariam, B., M. L. Brown, D. Deykin, and R. A. Cohen. 1990. Elevated glucose promotes generation of endothelium-derived vasoconstrictor prostanoids in rabbit aorta. J. Clin. Invest. 85:929-932.

36. Weidmann, P., C. Beretta-Piccolo, and B. N. Trost. 1985. Pressor factors and responsiveness in hypertension accompanying diabetes mellitus. Hypertension (Dallas). 7:II-33-42.

37. Weidmann, P., C. Beretta-Piccoli, G. Keusch, Z. Gluck, M. Mujagic, M. Grimm, A. Meier, and W. H. Ziegler. 1979. Sodium-volume factor cardiovascular reactivity and hypotensive mechanisms of diuretic therapy in hypertension associated with diabetes mellitus. Am. J. Med. 67:779-784.

38. Yue, D. K., S. McLennan, and J. R. Turtle. 1983. Non-enzymatic glycosylation of tissue protein in diabetes in the rat. Diabetologia. 24:377-381.

39. Vogt, B. W., E. D. Schleicher, and H. O. Wieland. 1982. Epsilon-aminolysine-bound glucose in human tissues obtained at autopsy: increase in diabetes mellitus. Diabetes. 31:1123-1127.

40. Aisaka, K., S. G. Gross, O. W. Griffith, and R. Levi. 1989. $N^{G}$-Methylarginine, an inhibitor of endothelium-derived nitric oxide synthesis, is a potent pressor agent in the guinea pig: does nitric oxide regulate blood pressure in vivo? Biochem. Biophys. Res. Commun. 160:881-886.

41. Rees, D. D., R. M. J. Palmer, and S. Moncada. 1989. Role of endothelium-derived nitric oxide in the regulation of blood pressure. Proc. Natl. Acad. Sci. USA. 86:3375-3378.

42. Vallance, P., J. Collier, and S. Moncada. 1989. Effects of endotheliumderived nitric oxide on peripheral arteriolar tone in man. Lancet. ii:997-1000.

43. Garthwaite, J., S. L. Charles, and R. Chess-Williams. 1988. Endotheliumderived relaxing factor release on activation of NMDA receptors suggests role as intercellular messenger in the brain. Nature (Lond.). 336:385-388.

44. Curran, R. D. T. R. Billiar, D. J. Stuehr, K. Hofmann, and R. L. Simmons. 1989. Hepatocytes produce nitrogen oxides from $L$-arginine in response to inflammatory products of Kupffer cells. J. Exp. Med. 170:1769-1774.

45. Garg, U. C., and A. Hassid. 1989. Inhibition of rat mesangial cell mitogenesis by nitric oxide generating agents. Am. J. Physiol. 257:F60-F66.

46. Brownlee, M., A. Cerami, and H. Vlassara. 1988. Advanced glycosylation endproducts in tissue and the biochemical basis of diabetic complications. $N$. Engl. J. Med. 318:1315-1321. 